\title{
Graphene Flakes in Arc Plasma: Conditions for the Fast Single-Layer Growth
}

\author{
Igor Levchenko $^{1 *}$, Uroš Cvelbar ${ }^{2 *}$, Michael Keidar ${ }^{3}$ \\ ${ }^{1}$ School of Chemistry, Physics, and Mechanical Engineering, Queensland University of Technology, Brisbane, \\ Australia \\ ${ }^{2}$ Jozef Stefan Institute, Ljubljana, Slovenia \\ ${ }^{3}$ George Washington University, Washington DC, USA \\ Email: Igor.Levchenko@qut.edu.au, "uros.cvelbar@ijs.si, keidar@gwu.edu
}

Received 19 January 2016; accepted 24 April 2016; published 27 April 2016

Copyright $(2016$ by authors and Scientific Research Publishing Inc.

This work is licensed under the Creative Commons Attribution International License (CC BY).

http://creativecommons.org/licenses/by/4.0/

(c) $\underset{\mathrm{EY}}{\mathrm{EY}}$ Open Access

\begin{abstract}
The results of systematic numerical studies of graphene flakes growth in low-temperature arc discharge plasmas are presented. Diffusion-based growth model was developed, verified using the previously published experiments, and used to investigate the principal effects of the process parameters such as plasma density, electron temperature, surface temperature and time of growth on the size and structure of the plasma-grown graphene flakes. It was demonstrated that the higher growth temperatures result in larger graphene flakes reaching $5 \mu \mathrm{m}$, and simultaneously, lead to much lower density of the carbon atoms adsorbed on the flake surface. The low density of the carbon adatoms reduces the probability of the additional graphene layer nucleation on surface of growing flake, thus eventually resulting in the synthesis of the most valuable single-layered graphenes.
\end{abstract}

\section{Keywords}

Graphene Flakes, Arc Plasma, Growth, Few-Layer Graphene

\section{Introduction}

Single- and few-layered graphene flakes demonstrate exceptional mechanical [1] [2], electrical [3], electronic [4] and magnetic properties [5] [6], and thus could be used as a platform for various advanced nanoscaled [7], microscaled [8] [9], and nano-electromechanical [10] systems and devices. Industrial wide-scale application of graphene in nanoelectronics requires development of methods capable of producing high-quality defect-free

\footnotetext{
"Corresponding author.
} 
flakes consisting of single or few graphene layers. Despite many recently developed production method such as mechanical exfoliation (cleavage) [11] [12], metal precipitation [13] and chemical vapour deposition [14], the large-scaled fabrication of the single- and few-layered graphene is still a challenge [15] [16].

Plasma-based techniques are the recently developed methods capable of producing the nanoscaled materials with quite different properties [17] [18]. Among others, the arc-discharge method is promising for producing graphene-containing materials in near-industrial scale [19] [20]. The arc-produced graphene flakes usually feature several (up to 5 - 10) layers and size up to ten micrometers, which is suitable for nanoelectronic applications [21]. In the recently developed process, few-layer graphene flakes are synthesized using a high-current arc discharge at a relatively high gas pressure of 0.5 to $1 \mathrm{~atm}$. (see scheme and photo in Figure 1). Both cathode and anode are made of carbon rods of 5 - $10 \mathrm{~mm}$ dia. Depending on the process, anode can be made with a hole in the center where the mixture of graphitic carbon catalyst (i.e., Y-Ni powder) is placed. The anode and cathode materials are the source of carbon. Optionally, a magnetic system can be used to generate a non-uniform magnetic field $(\approx 0.1 \mathrm{~T})$ in the process zone [22]. As the main plasma-generating gas the helium at a pressure $0.5 \mathrm{~atm}$. can be used [23] [24]. More details on the process and characterization of the produced graphene flakes can be found in our recent publications [25]. Carbonous deposit is then removed from the collecting surface, sonicated to separate amorphous carbon and graphene flakes (Figure 1(b)), and the ready graphene-containing solution is prepared (Figure 1(c)) which can be used for e.g. the fabrication of conductive inks (Figure 1(d)).

The growth process and hence the properties of the prepared graphene flakes significantly depend on the plasma and growth parameters (plasma density, electron energy, surface temperature etc.). Numerous experimental works were conducted and the results are published, but a comprehensive picture of the graphene growth still remains unclear, despite of the many applied efforts. Here we present the results of numerical studies of the

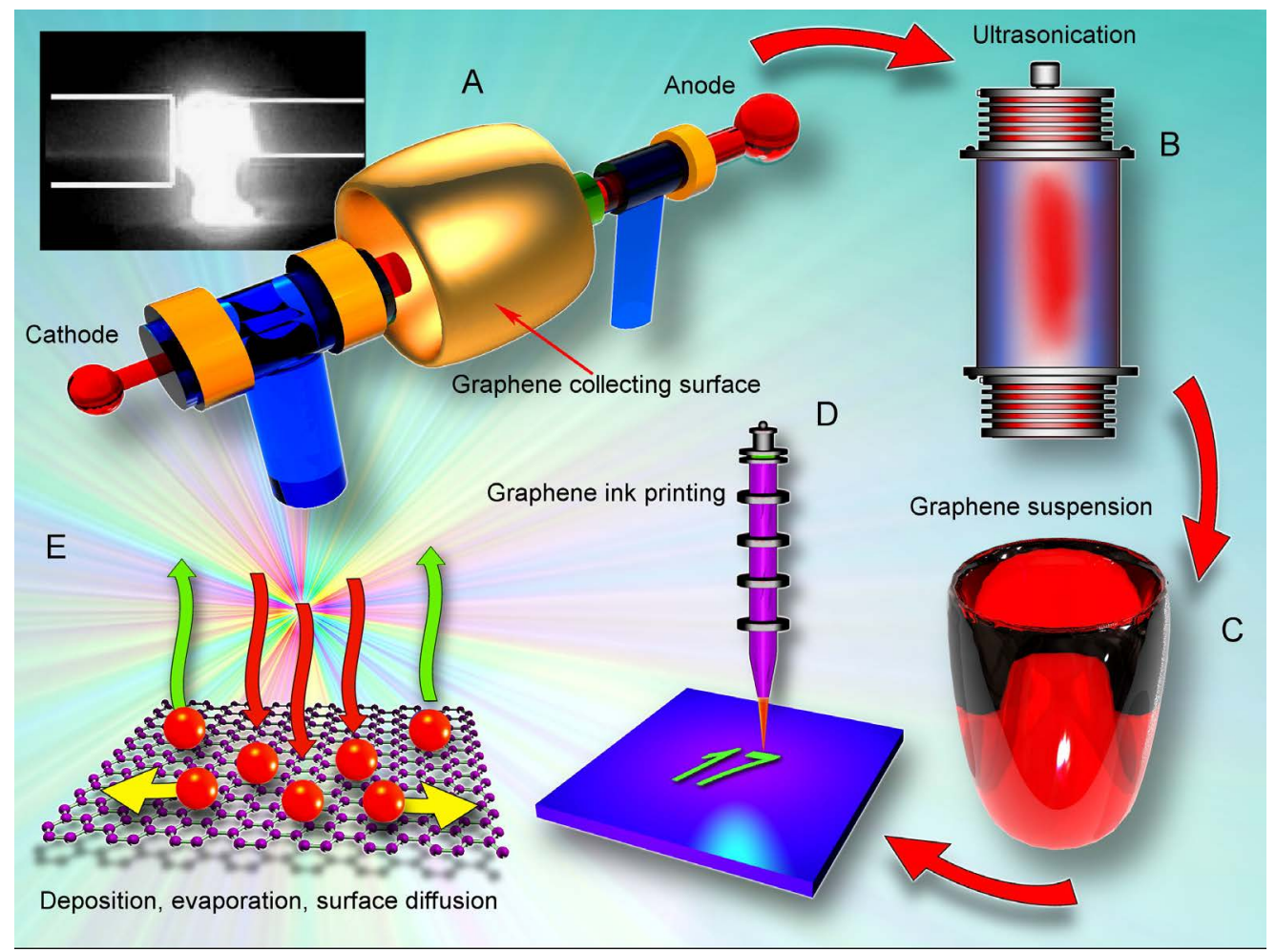

Figure 1. Schematics of the process of graphene flake fabrication in arc discharge setup. (A) Graphene growth apparatus consisting of anode, cathode, graphene collecting surface and (optionally) focusing magnet. DC discharge (30 - $120 \mathrm{~V}, 25$ $100 \mathrm{~A}$ ) is sustained in He gas at pressure of $0.5-1.0 \mathrm{~atm}$. Inset is the discharge photo. Carbon material is supplied due to anode evaporation; (B) The carbonous deposit is removed from the surface and ultrasonicated, and eventually (C) the ready graphene flake suspension is produced which can be used, e.g., for printing (D); Schematic of the processes taken into account during the numerical simulation (E): carbon atoms influx from plasma to the growing graphene flake, evaporation of carbon atoms from the graphene surface, surface diffusion of carbon atoms around the graphene surface, and incorporation of carbon adatoms to the edges of flakes. 
graphene flake growth in arc plasmas, conducted to mainly determine the general effect of the plasma parameters on the graphene growth behavior and specifically, to find the process parameters which directly affect the growth rates and nucleation conditions, with an ultimate view to better understand the processes leading to the transition from the formation of single-layer graphenes to few-layered flakes. We use here a relatively simple diffusion-based model which is not time-consuming, as compared with the molecular dynamics or Monte-Carlo techniques, but still is capable to reveal the main characteristics and parameters.

\section{Model and Results}

\subsection{Model Formulation}

The main processes taken into account are illustrated in Figure 1(e). Specifically, we consider the three main processes: 1) carbon atom influx from plasma to surface of growing graphene flake; 2) evaporation of adsorbed atoms (adatoms) from the graphene surface to plasma; and 3) surface diffusion of adatoms about the graphene surface, followed by incorporation into the graphene flake structure at the edges; the last process ensures the growth of the flake. Thus, the balance equation for the adatom density $n_{c}$ on graphene surface in plasma is:

$$
\frac{\mathrm{d} n_{c}}{\mathrm{~d} t}=\Psi_{\downarrow}-\Psi_{\uparrow}-\Psi_{\rightarrow \text { out }}
$$

where $\Psi_{\downarrow}=n_{p} V_{B}$ is the density change due to influx from plasma (only flux of ions with the energy sufficient to establish bonds with the growing graphene flake is taken into account), here $V_{B}=\sqrt{\kappa T_{e} / m_{i}}$ is the Bohm velocity; $\varepsilon_{e}$ is the electron energy; $e$ is the electron charge, $k$ is the Boltzmann's constant, and $m_{i}$ is the ion mass; $\Psi_{\uparrow}=n_{c} v_{a}$ is the adatom density change due to evaporation from the graphene flake surface; here $v_{a}=v_{0} \mathrm{e}^{-\frac{e \varepsilon_{a}}{k T_{s}}}, T_{s}$ is the graphene flake surface temperature, $v_{0}=\frac{2 \kappa T_{s}}{h}$ is the lattice vibration frequency, $h$ is the Plank's constant, and $\varepsilon_{a}$ is the energy of carbon adatom evaporation.

The diffusion flux from the flake side to edges $\Psi_{\rightarrow \text { out }}$ can be found by considering the diffusion flux of adatoms from the flake edge, and for the flake with radius $r_{1}$ one can obtain:

$$
\Psi_{\rightarrow \text { out }}=\frac{2 \sqrt{n_{c}}}{r_{1}} v_{0} \mathrm{e}^{-\frac{\varepsilon_{d}}{k T_{s}}}
$$

where $\varepsilon_{d}$ is the surface diffusion activation energy. Finally we obtain:

$$
\frac{\mathrm{d} n_{c}}{\mathrm{~d} t}=n_{p} \sqrt{\frac{e \varepsilon_{e}}{m_{i}}}-\frac{2 k T_{s}}{h}\left(n_{c} \mathrm{e}^{-\frac{e \varepsilon_{a}}{k T_{s}}}+\frac{2 \sqrt{n_{c}}}{r_{1}} \mathrm{e}^{-\frac{\varepsilon_{d}}{k T_{s}}}\right)
$$

Equation (4) is required for the two aims, namely 1) to find the diffusion flux from the flake side to the external edges $\Psi_{\rightarrow \text { out }}$ which determines the flake growth rate due to adatoms attachment to edges, and 2) to estimate the nucleation of new flake on the surface of growing graphene, i.e. transition from single-layer to the two-layer (and further to few-layer) graphene flake. With the adatom density $n_{c}$ calculated from (1), the graphene growth rate can be estimated as:

$$
\frac{\mathrm{d} r_{1}}{\mathrm{~d} t}=\frac{r_{2}}{2} \lambda^{2} \Psi_{\rightarrow \text { out }}, \mathrm{m} \cdot \mathrm{s}^{-1}
$$

where $\lambda$ is the mean (effective) graphene lattice period which was calculated from the mean surface density of graphene being $3.82 \times 10^{15} \mathrm{~cm}^{-2}$ [26]. We will also assume that the graphene flake is in the thermodynamic equilibrium with the plasma, and hence the graphene flake temperature $T_{s}$ is equal to the gas temperature $T_{g}$.

The task becomes more complicated after the nucleation of a second flake on the surface of a single-layered graphene. In this case the growth rate of the second layer is described by

$$
\frac{\mathrm{d} r_{2}}{\mathrm{~d} t}=\frac{r_{2}}{2} \lambda^{2}\left(\Psi_{\rightarrow \text { out }}^{\prime}+\Psi_{\rightarrow \text { in }}\right), \mathrm{m} \cdot \mathrm{s}^{-1}
$$

where $r_{2}$ is the second flake radius, $\Psi_{\rightarrow \text { out }}^{\prime}$ is the diffusion flux from the second flake to the external edges, and $\Psi_{\rightarrow \text { in }}$ is the diffusion flux from the surface of first flake (substrate) to the edges of the second flake. 


\subsection{Numerical Results I: Growth of Single-Layer Graphene Flake}

Figure 2 illustrates the calculated dependencies of the carbon adatom density on the surface of growing graphene flake, as well as single-layer flake size on growth time with electron temperature, plasma density, surface temperature and temperature profile as parameters.

The plasma parameters and growth conditions used for the simulations are typical for the arc-based process effective for graphene and carbon nanotube growth [27] [28]. Importantly, the gas temperature in arc discharge is close to the optimum values of 1000 - $1300 \mathrm{~K}$ [29]. The two main process activation energies namely the surface diffusion activation energy $\varepsilon_{d}$ and carbon evaporation energy $\varepsilon_{a}$ strongly influence the growth process. Direct measurement of these values is a hard task, thus we used the results of $a b$ initio calculations [30] for $\varepsilon_{a}$ energy and have verified the most important diffusion activation energy $\varepsilon_{d}$ by our previous experimental works [19] [23]. The typical growth time was $1 \mathrm{~ms}$, determined from the characteristic velocities $\left(10-100 \mathrm{~m} \cdot \mathrm{s}^{-1}\right) \mathrm{in}$ arc plasma and inter-electrode gap of several $\mathrm{mm}$.
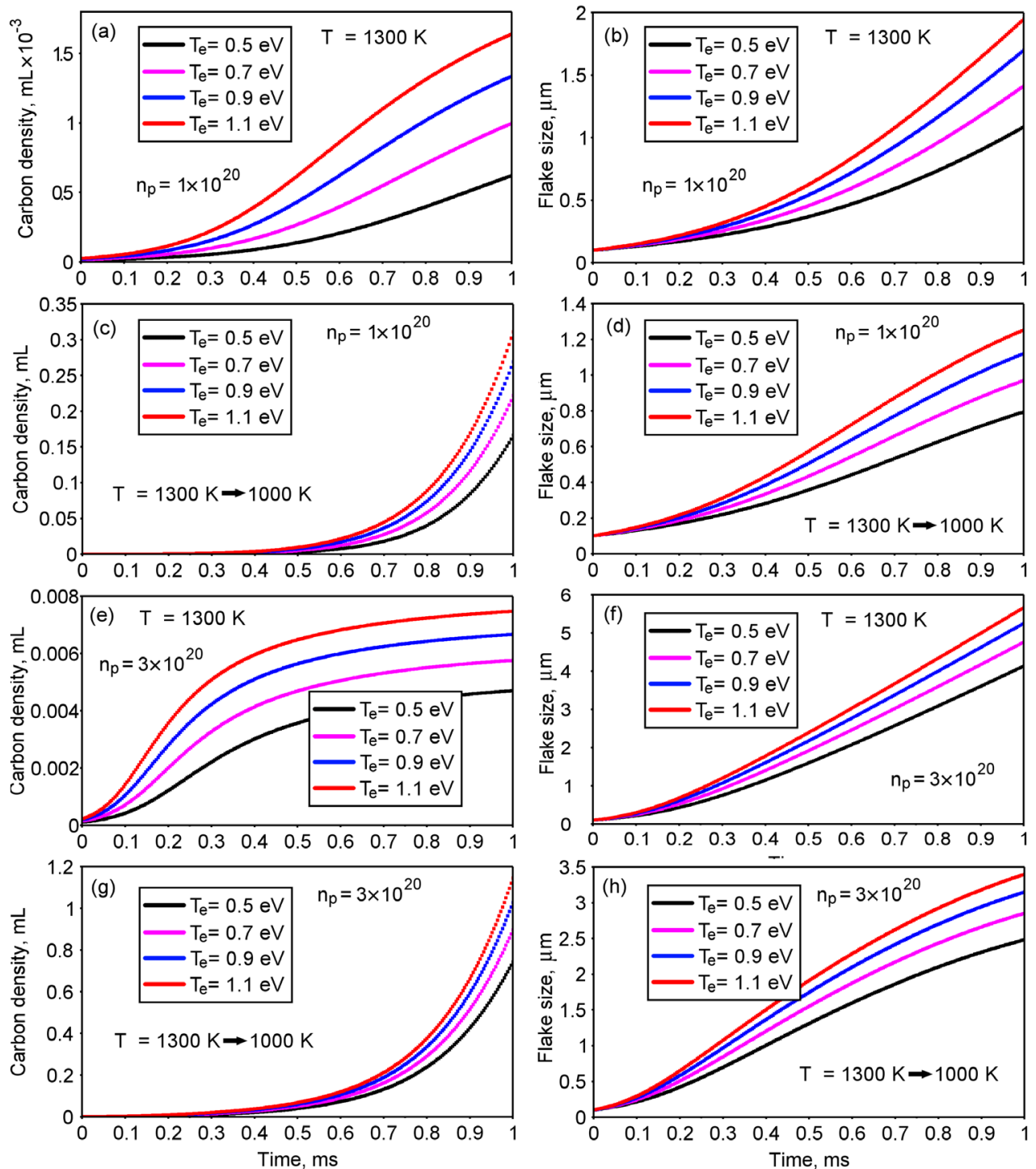

Figure 2. Dependencies of carbon adatom density on the surface of growing graphene flake (left subpanel-(a), (c), (e), (g)) and monolayer flake size (right subpanel-(b), (d), (f), (h)) on the growth time with electron temperature, plasma density, temperature and temperature profile as parameters. The growth under the constant temperature condition results in larger graphene flakes and lower density of adatoms on the graphene surface, which ensures the formation of single-layered graphenes. Electron energy could also be used as a control factor to influence the graphene structure. 
Figures 2(a)-(d) represent the calculated dependencies of the carbon adatom density on the surface of growing graphene flake and single-layered flake size on the growth time with electron temperature ( 0.5 to $1.1 \mathrm{eV}$ ), surface temperature and temperature profile as parameters, for the lowest considered plasma density of $10^{20} \mathrm{~m}^{-3}$. Figure 2(a) and Figure 2(b) show these dependencies for the constant surface temperature of $1300 \mathrm{~K}$, whereas the graphs shown on Figure 2(c) and Figure 2(d) are the results for the surface temperature changing from 1300 $\mathrm{K}$ at the initial time moment (nucleation) down to $1000 \mathrm{~K}$. These two cases conditionally represent the two possible particle (growing flake) trajectories in plasma: the first one corresponds to the flake growing in the hot plasma jet, and the second one corresponds to the flakes which quickly abandon the plasma; as a rule, these particles are deposited onto different areas of the collecting surfaces as demonstrated by our experiments [19]. Apparently, these two cases illustrated the limiting conditions useful to mark the outermost growth conditions and structures; the most quantity of the graphene is growing in the mean conditions.

Comparing the graphs in Figure 2(a) and Figure 2(c) (time dependence of the adatom density on growing flake) one can see that the density is very low $\left(\approx 10^{-3}\right.$ of monolayer density) for the case of a constant temperature $1300 \mathrm{~K}$ (Figure 2(a)), thus making the probability of new layer nucleation very low. In contrast, the density sharply increases for the second case (temperature changing from 1300 to $1000 \mathrm{~K}$ during the growth, Figure 2(c)) and reaches the relative density 0.3 (i.e., one adatom per three surface atoms) which could be considered as quite favorable for nucleation of new layer and hence, transition from the single-layered to the two-layered flake structure. The adatom density also strongly depends on the plasma electron density (due to the effect on the Bohm velocity), this reveals one more handle to control the process by plasma parameters.

The time dependencies of the single-layer flake sizes are presented in Figure 2(b) and Figure 2(d). The maximum flake diameters reach 2 and $1.25 \mu \mathrm{m}$ for the first and second cases, respectively. In spite of this apparent similarity, the difference in the processes is quite significant due to the very different adatom densities on the flake surfaces which can result in the formation of a multi-layered flake in the case of decreasing growth temperature (Figure 2(b)), but the graphene flake grown at the constant temperature of $1300 \mathrm{~K}$ (Figure 2(d)) will definitely remain single-layered. The plasma electron density also influences the flake size but not so strongly: the sizes of flakes grown in the plasma with the electron energies at 0.5 and $1.1 \mathrm{eV}$ differ by the factor of 2, whereas the adatom densities on flakes growing under the same conditions differ by the factor of 3 ; this could be explained by more intense evaporation of adatoms from the graphene surface at higher temperatures. The larger size of the flakes grown at the constant temperature could be attributed to higher diffusion fluxes to the flake edges, which are responsible for the graphene growth according to (5). It also could be noted that the graphene growth rates steady increase with the time when the process is conducted at the constant temperature, whereas the second case (graphene surface temperature decreases to $1000 \mathrm{~K}$ ) demonstrates an evident saturation.

Hence, one can derive that the process at the high constant temperature features at least two significant advantages, namely 1) larger graphene flake size and even more important, 2) very low density of adatom on the surface of growing graphene which results in the formation of single-layered flakes.

Similar dependencies are shown in Figures 2(e)-(h) for the higher plasma density of $3 \times 10^{20} \mathrm{~m}^{-3}$. In this case the graphene flakes reach $6 \mu \mathrm{m}$ for the constant temperature case and $3.5 \mu \mathrm{m}$ for the growth temperatures descending to $1000 \mathrm{~K}$. The influence of the plasma electron energy is not so strong at this higher plasma density. The adatom density on graphenes grown at the constant temperatures still remains low $\left(8 \times 10^{-3} \mathrm{~mL}\right)$, thus demonstrating the possibility to grow large $(6 \mu \mathrm{m})$ single-layered flakes. It could be also mentioned that the adatom density demonstrate saturation with time, i.e. the flake could grow further in the single-layer mode.

Importantly, the conclusion about better growth under the constant temperature conditions was confirmed with numerous experiments with the magnetic-enhanced arc setups, where the permanent magnet was used to focus plasma and elevate the electron temperature. Indeed, higher yield and better quality of the deposit was obtained in the magnetic-enhanced arc discharges.

In Figure 3 we present the dependencies of the carbon adatom density on graphene surface and flake size on the growth time with electron temperature, plasma density and temperature as parameters. These dependencies are similar to those in Figure 2, but calculated for lower surface temperature $T_{\mathrm{s}}=1000 \mathrm{~K}$. In this case the increase of the plasma density is not efficient. It can indeed ensure larger flake size (1.6 $\mu \mathrm{m}$ for the plasma density of $2 \times 10^{20} \mathrm{~m}^{-3}$ versus $1.0 \mu \mathrm{m}$ for $1 \times 10^{20} \mathrm{~m}^{-3}$ ) but at the expense of very high adatom density on the graphene surface (reaching 1, i.e. complete coverage of the graphene surface with adatom), which unavoidable resulted in the nucleation of new layer. Hence, it is impossible to grow relatively large (up to several $\mu \mathrm{m}$ ) graphene flakes at low temperatures. Indeed, low plasma densities could sustain the growth in the single-player mode 

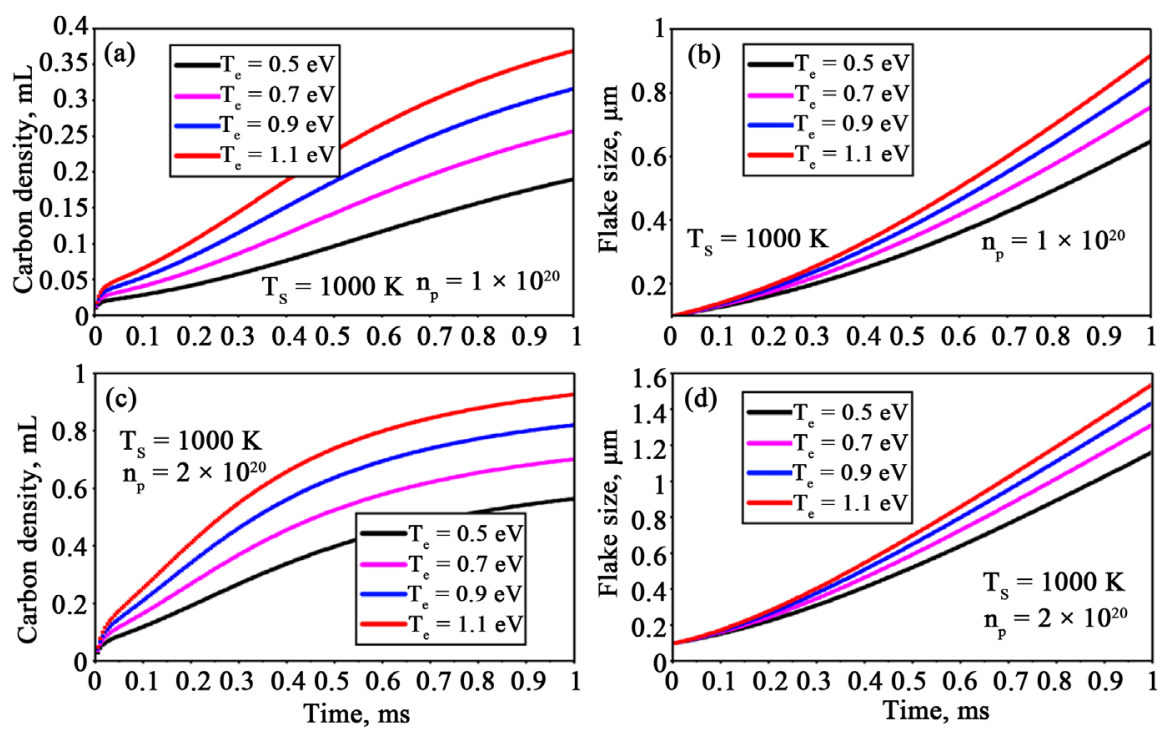

Figure 3. Dependencies of the carbon adatom density on the surface of growing graphene flake (left subpanel-(a), (c)) and single-layer flake size (right subpanel-(b), (d)) on the growth time with electron temperature, plasma density and temperature as parameters. Under the same conditions and relatively low graphene surface temperature $(1000 \mathrm{~K})$, the increase of the plasma density is not efficient and could ensure larger flake size at the expense of very high adatom density on the graphene surface, which unavoidable resulted in the nucleation of new layer.

(i.e., at low adatom density), but the size of the graphene flake in this case does not exceed $1 \mu \mathrm{m}$; when higher plasma density is used, the adatom density significantly increases and unavoidably leads to the transition to multi-layer graphene which is not favorable. The reasons for this are low diffusion fluxes to the graphene edges which promote the flake growth; on the other hand, low evaporation leads to the fast accumulation of the adatoms and hence, nucleation of new layers. The increase of the electron energy in plasma cannot help in this case, since it does not influence the diffusion and the graphene growth rates. Based on these results one can conclude that the low-temperature growth is not favorable and the low growth rates cannot be compensated by higher plasma density without compromising the graphene quality due to the transition to the multi-layered growth mode.

Moreover the calculations for elevated surface temperature of $1200 \mathrm{~K}$ and much higher plasma densities (5 ... $20 \times 10^{20} \mathrm{~m}^{-3}$ ) are presented in Figure 4. The dependencies of the carbon adatom density on the surface and single-layer flake size on the growth time with electron temperature and plasma density as parameters are quite different of those shown in Figure 3. Indeed, the higher graphene surface temperature of $1200 \mathrm{~K}$ and significantly higher plasma densities resulted in the formation very large $\left(6 \mu \mathrm{m}\right.$ for $5 \times 10^{20} \mathrm{~m}^{-3}$ and $14 \mu \mathrm{m}$ for $20 \times$ $10^{20} \mathrm{~m}^{-3}$ ) graphenes at the relatively low adatom density on the graphene surface (not exceeding $0.06 \mathrm{~mL}$ for $5 \times$ $10^{20} \mathrm{~m}^{-3}$ and $0.25 \mathrm{~mL}$ for $20 \times 10^{20} \mathrm{~m}^{-3}$ ). Despite a very high plasma density, these relatively low adatom densities definitely ensure the graphene growth in the single-layer mode, since the formation of new graphene layers is impossible at such conditions. The apparent reasons for this are significantly enhanced surface diffusion rate at higher temperatures (since the surface diffusion exponentially depends on the temperature) and higher evaporation from the surface which prevents the accumulation of the excessive adatoms which could cause nucleation of a new layer. Much higher atom fluxes from the dense $\left(20 \times 10^{20} \mathrm{~m}^{-3}\right)$ plasma lead to the strong diffusion to the graphene edges where adatoms incorporate in the graphene structure. We can also note that the influence of the electron energy $\varepsilon_{e}$ on the graphene size is low (since the Bohm velocity does not influence the diffusion), but the adatom density strongly depends on $\varepsilon_{e}$ and hence could be efficiently controlled by changing, i.e., discharge voltage or using magnetic field to enhance ionization and electron energy. Another important fact is the apparent saturation of the adatom densities in both $\left(5 \times 10^{20} \mathrm{~m}^{-3}\right.$ and $\left.20 \times 10^{20} \mathrm{~m}^{-3}\right)$ cases, which means that the further growth is possible in the single-layer mode by extending the growth zone; in this case the measures should be taken to maintain the plasma and graphene flake temperatures at the higher enough level, to avoid the situation shown in Figure 3 (very high adatom density at low temperatures leading the transition to multi-layered growth mode). An extended magnetic field capable to maintain very dense plasma may help in this case. Near-linear 
dependence of the graphene flake size on time is also an important effect which was not noticed under condition described in Figure 2 and Figure 4.

To better characterize growth of graphenes in plasma, we have calculated the dependencies of the single-layer flake size on electron temperature in plasma, with the plasma density and surface temperature as parameters (Figure 5). From this figure one can see that these dependencies are relatively weak and cannot be used for efficient increase of the flake sizes. On the other hand, the nucleation of new layers cold be controlled by the electron energy (Figure 4), and hence this parameter is important for maintaining the most desirable single-layered
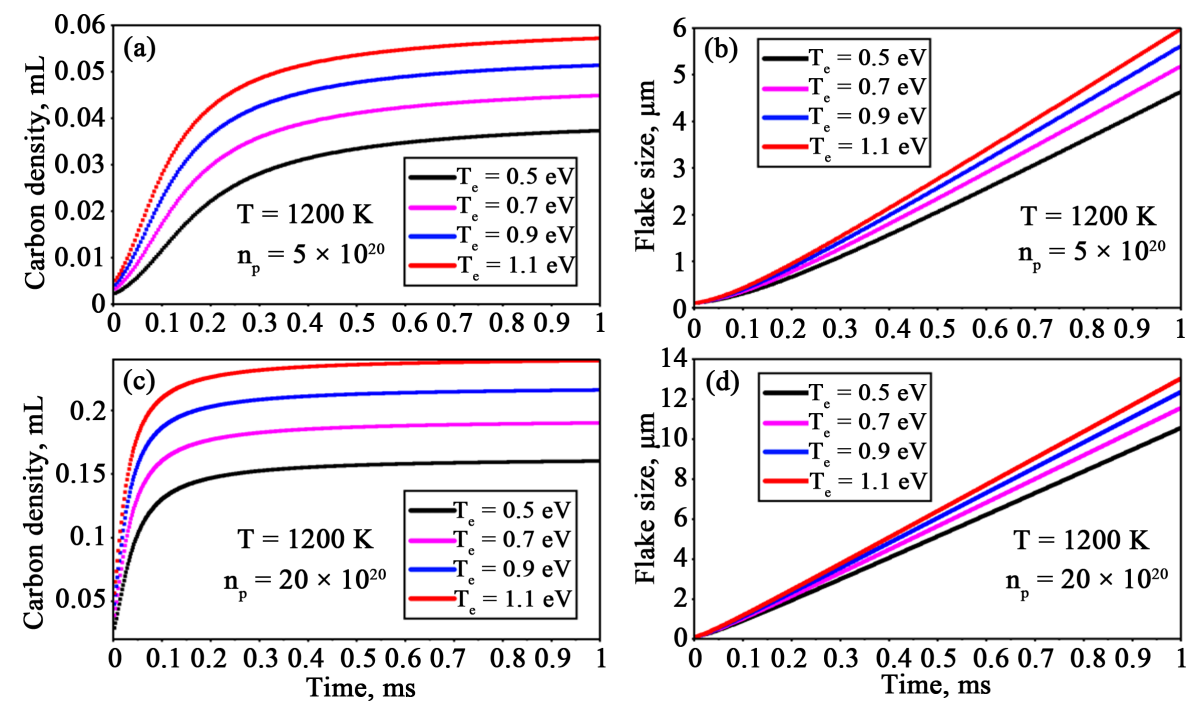

Figure 4. Dependencies of the carbon adatom density on the surface of growing graphene flake (left subpanel-(a), (c)) and single-layer flake size (right subpanel-(b), (d)) on the growth time with electron temperature, plasma density and temperature as parameters. Higher graphene surface temperature $(1200 \mathrm{~K})$ and significantly higher plasma densities are quite efficient for the fabrication of large flakes at the relatively low adatom density on the graphene surface. Despite a very high plasma density, relatively low adatom densities are maintained and the formation of new graphene layers is impossible.
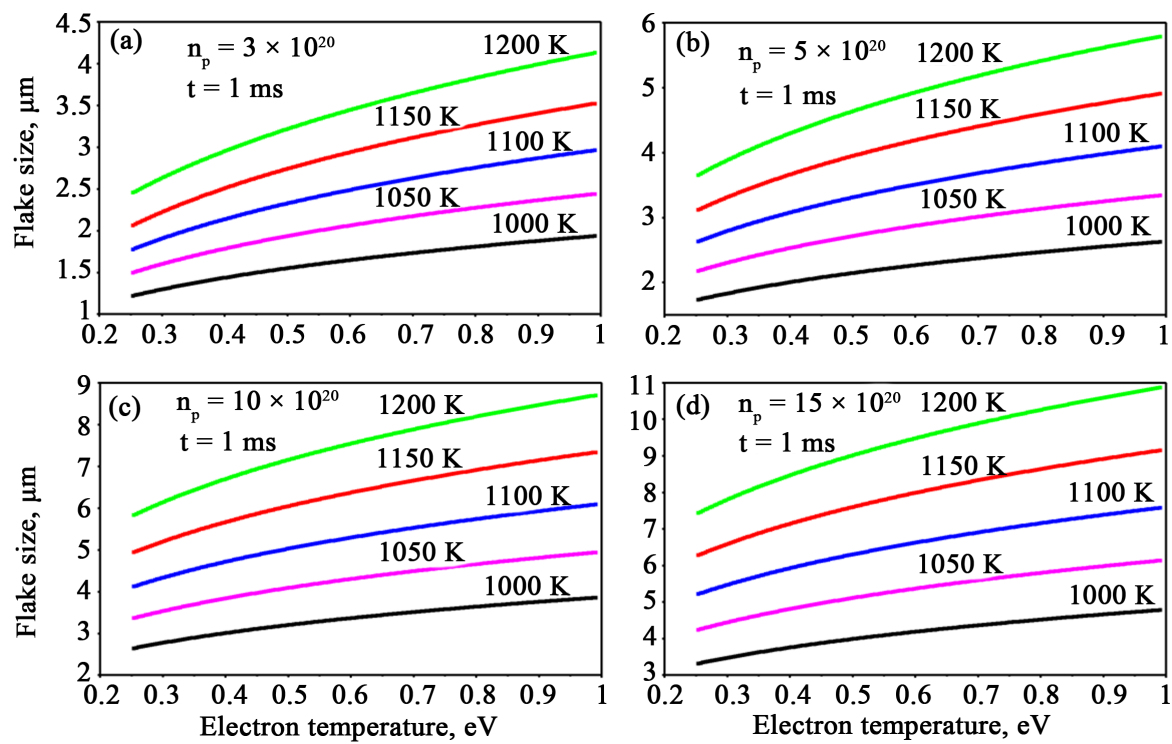

Figure 5. Dependencies of the single-layer flake size on electron temperature in plasma, with the plasma density and surface temperature as parameters. These dependencies are relatively weak and cannot be recommended for increasing the flake sizes, whereas they are important for maintaining the single-layered growth mode. We use here a growth time of $1 \mathrm{~ms}$ which is typical to small discharge gap. In other conditions the typical residence time might be larger than $1 \mathrm{~ms}$, up to tens of ms; in this case the size of graphene will be accordingly larger. 
growth mode. The surface temperature appears to be an important parameter for the growth control, as seen in Figure 5. It can be stressed that the small graphene flakes are usually in a thermodynamic equilibrium with the plasma and process gas, and thus the graphene surface control is reduced to maintaining the proper plasma parameters.

Importantly, graphene flake growth rates do not demonstrate significant saturation with time, except of the situation when the surface temperature drops off abruptly (Figure 2(d) and Figure 2(h)). Thus, the processes ensuring longer growth under the high enough temperatures and ion fluxes could result in much larger graphenes which are required for many applications.

\section{Summary}

We have demonstrated that the plasma parameters such as gas temperature, plasma density and electron energy could be efficiently used for maintaining the graphene growth to relatively large (up to $10 \mu \mathrm{m}$ ) sizes in the single layer mode. High growth rates and large flakes could be synthesized in plasma of $5 \times 10^{20} \mathrm{~m}^{-3}$ to $20 \times 10^{20} \mathrm{~m}^{-3}$ density, but the graphene surface temperature should be at least 200 to $1300 \mathrm{~K}$ to avoid accumulation of the excessive adatoms on graphene surface and hence, avoid the nucleation of new layers which causes transition to the undesired multilayer growth mode. The further works should study in detail the process and kinetics of the layers formation, and to find the optimum conditions for the production of the defect-free graphene flakes.

\section{Acknowledgements}

I. L. acknowledges the support from the School of Chemistry, Physics and Mechanical Engineering, Science and Engineering Faculty, Queensland University of Technology. This work was partially supported by Slovenian Research Agency (ARRS), project L2-6769. Work at GWU was sponsored in part by the GW Institute for Nanotechnology.

\section{References}

[1] Frank, I.W., Tanenbaum, D.M., van der Zande, A.M. and McEuen, P.L. (2007) Mechanical Properties of Suspended Graphene Sheets. Journal of Vacuum Science and Technology B, 25, 2558-2561. http://dx.doi.org/10.1116/1.2789446

[2] Cohen-Tanugi, D. and Grossman, J.C. (2014) Mechanical Strength of Nanoporous Graphene as a Desalination Membrane. Nano Letters, 14, 6171-6178. http://dx.doi.org/10.1021/nl502399y

[3] Peres, N.M.R., Guinea, F. and Castro Neto, A.H. (2006) Electronic Properties of Disordered Two Dimensional Carbon. Physical Review B, 73, Article ID: 125411. http://dx.doi.org/10.1103/physrevb.73.125411

[4] Dedkov, Y.S. and Fonin, M. (2010) Electronic and Magnetic Properties of the Graphene-Ferromagnet Interface. New Journal of Physics, 12, Article ID: 125004. http://dx.doi.org/10.1088/1367-2630/12/12/125004

[5] Wang, Z., Tang, C., Sachs, R., Barlas, Y. and Shi, J. (2015) Proximity-Induced Ferromagnetism in Graphene Revealed by the Anomalous Hall Effect. Physical Review Letters, 114, Article ID: 016603. http://dx.doi.org/10.1103/physrevlett.114.016603

[6] Levchenko, I., Keidar, M., Xu, S., Kersten, H. and Ostrikov, K. (2013) Low-Temperature Plasmas in Carbon Nanostructure Synthesis. Journal of Vacuum Science and Technology B, 31, Article ID: 050801.

[7] Craighead, H.G. (2000) Nanoelectromechanical Systems. Science, 290, 1532-1536. http://dx.doi.org/10.1126/science.290.5496.1532

[8] Bunch, J.S., van der Zande, A.M., Verbridge, S.S., Frank, I.W., Tanenbaum, D.M., Parpia, J.M., Craighead, H.G. and McEuen, P.L. (2007) Electromechanical Resonators from Graphene Sheets. Science, 315, 490-493. http://dx.doi.org/10.1126/science.1136836

[9] Veen, J., Gomez, A., van der Zant, H. and Steele, G. (2013) Flexible Graphene Devices with an Embedded Back-Gate. Graphene, 2, 13-17. http://dx.doi.org/10.4236/graphene.2013.21003

[10] Ekinci, K.L. and Roukes, M.L. (2005) Nanoelectromechanical Systems. Review if Scientific Instrument, 76, Article ID: 061101. http://dx.doi.org/10.1063/1.1927327

[11] Geim, A.K. and Novoselov, K.S. (2007) The Rise of Graphene. Nature Materials, 6, 183-191. http://dx.doi.org/10.1038/nmat1849

[12] Torres, L., Gomez Armas, L. and Carlos Seabra, A. (2014) Optimization of Micromechanical Cleavage Technique of Natural Graphite by Chemical Treatment. Graphene, 3, 1-5. http://dx.doi.org/10.4236/graphene.2014.31001

[13] Dahal, A. and Batzill, M. (2014) Graphene-Nickel Interfaces: A Review. Nanoscale, 6, 2548-2562. 
http://dx.doi.org/10.1039/c3nr05279f

[14] Deokar, G., Avila, J., Razado-Colambo, I., Codron, J.-L., Boyaval, C., Galopin, E., et.al. (2015) Towards High Quality CVD Graphene Growth and Transfer. Carbon, 89, 82-92. http://dx.doi.org/10.1016/j.carbon.2015.03.017

[15] Levchenko, I., Ostrikov, K., Zheng, J., Li, X., Keidard, M. and Teo, K. (2016) Scalable Graphene Production: Perspectives and Challenges of Plasma Applications. Nanoscale, in Press. http://dx.doi.org/10.1039/C5NR06537B

[16] Vlassiouk, I., Polizos, G., Cooper, R., Ivanov, I., Keum, J.K., Paulauskas, F., Datskos, P. and Smirnov, S. (2015) Strong and Electrically Conductive Graphene-Based Composite Fibers and Laminates. ACS Applied Materials and Interfaces, 7, 10702-10709. http://dx.doi.org/10.1021/acsami.5b01367

[17] Xu, S., Huang, S.Y., Levchenko, I., Zhou, H.P., Wei, D.Y., Xiao, S.Q., et al. (2011) Highly Efficient Silicon Nanoarray Solar Cells by a Single-Step Plasma-Based Process. Advanced Energy Materials, 1, 373-376. http://dx.doi.org/10.1002/aenm.201100085

[18] Levchenko, I., Romanov, M. and Keidar, M. (2003) Investigation of a Steady-State Cylindrical Magnetron Discharge for Plasma Immersion Treatment. Journal of Applied Physics, 94, 1408-1413. http://dx.doi.org/10.1063/1.1590054

[19] Park, H., Noh, S.H., Lee, J.H., Lee, W.J., Jaung, J.Y., Lee, S.G. and Han, T.H. (2015) Large Scale Synthesis and Light Emitting Fibers of Tailor-Made Graphene Quantum Dots. Scientific Reports, 5, Article ID: 14163. http://dx.doi.org/10.1038/srep14163

[20] Volotskova, O., Levchenko, I., Shashurin, A., Raitses, Y., Ostrikov, K. and Keidar, M. (2010) Single-Step Synthesis and Magnetic Separation of Graphene and Carbon Nanotubes in Arc Discharge Plasmas. Nanoscale, 2, 2281-2285. http://dx.doi.org/10.1039/c0nr00416b

[21] Keidar, M., Shashurin, A., Li, J., Volotskova, O., Kundrapu, M. and Zhuang, T. (2011) Arc Plasma Synthesis of Carbon Nanostructures: Where Is the Frontier? Journal of Physics D: Applied Physics, 44, Article ID: 174006.

[22] Keidar, M., Levchenko, I., Arbel, T., Alexander, M., Waas, A.M. and Ostrikov, K. (2008) Magnetic-Field-Enhanced Synthesis of Single-Wall Carbon Nanotubes in Arc Discharge. Journal of Applied Physics, 103, Article ID: 094318. http://dx.doi.org/10.1063/1.2919712

[23] Levchenko, I., Volotskova, O., Shashurin, A., Raitses, Y., Ostrikov, K. and Keidar, M. (2010) The Large-Scale Production of Graphene Flakes Using Magnetically-Enhanced Arc Discharge between Carbon Electrodes. Carbon, 48, 4570-4574. http://dx.doi.org/10.1016/j.carbon.2010.07.055

[24] Levchenko, I., Ostrikov, K., Mariotti, D. and Švrček, V. (2009) Self-Organized Carbon Connections between Catalyst Particles on a Silicon Surface Exposed to Atmospheric-Pressure $\mathrm{Ar}+\mathrm{CH}_{4}$ Microplasmas. Carbon, 47, 2379-2390. http://dx.doi.org/10.1016/j.carbon.2009.04.031

[25] Fang, X., Donahue, J., Shashurin, A. and Keidar, M. (2015) Plasma-Based Graphene Functionalization in Glow Discharge. Graphene, 4, 1-6. http://dx.doi.org/10.4236/graphene.2015.41001

[26] Pop, E., Varshney, V. and Roy, A.K. (2012) Thermal Properties of Graphene: Fundamentals and Applications. MRS Bulletin, 37, 1273-1281. http://dx.doi.org/10.1557/mrs.2012.203

[27] Keidar, M., Waas, A.M., Raitses, Y. and Waldorff, E. (2006) Modeling of the Anodic Arc Discharge and Conditions for Single-Wall Carbon Nanotube Growth. Journal of Nanoscience and Nanotechnology, 6, 1309-1314. http://dx.doi.org/10.1166/jnn.2006.159

[28] Shashurin, A., Keidar, M. and Beilis, I.I. (2008) Voltage-Current Characteristics of an Anodic Arc Producing Carbon Nanotubes. Journal of Applied Physics, 104, Article ID: 063311. http://dx.doi.org/10.1063/1.2986572

[29] Fang, X., Shashurin, A. and Keidar, M. (2015) Role of Substrate Temperature at Graphene Synthesis in an Arc Discharge. Journal of Applied Physics, 118, Article ID: 103304.

[30] Lee, Y.H., Kim, S.G. and Tománek, D. (1997) Catalytic Growth of Single-Wall Carbon Nanotubes. Physical Review Letter, 78, 2393-2396. http://dx.doi.org/10.1103/PhysRevLett.78.2393 